La devaluación y sus efectos composicionales sobre el set de importaciones

\author{
Andrés César \\ Tesis de Maestría \\ Maestría en Economía \\ Universidad Nacional de La Plata
}

Directora: Irene Brambilla

17 de Octubre de 2014 


\title{
La devaluación y sus efectos composicionales sobre el set de importaciones*
}

\author{
Andrés César ${ }^{\dagger}$
}

\begin{abstract}
Resumen
Este trabajo pretende aportar a la comprensión del fenómeno del traspaso a precios domésticos de las devaluaciones del tipo de cambio, o exchange rate pass-through. Se realiza un análisis econométrico basado en datos correspondientes al período de crisis Argentina (1999-2004) que tuvo su epicentro en los años 2001-2002. Se intenta evaluar si los cambios en el set de importaciones son relevantes para amortiguar el traslado de aumentos en el precio de monedas extranjeras a los precios de venta de productos importados en el mercado doméstico. En este sentido, se estudia la racionalidad de dicho canal y su relevancia empírica durante la crisis cambiaria que atravesó Argentina y se trata de evaluar si efectivamente el precio en dólares de los productos importados cayó; si existieron cambios composicionales en la canasta de importación; y de ser así, si la sustitución fue en favor de variedades más económicas y/o de menor calidad.
\end{abstract}

\begin{abstract}
This paper aims to contribute to understanding the phenomenon of exchange rate pass-through into import prices. The econometric analysis is based on data of the Argentine crisis period (1999-2004) with its epicenter in the years 2001-2002. It tries to assess whether changes in the set of imports are relevant to dampen the exchange rate pass-through at the sales prices of imported products in the domestic market. In this sense, I study the rationality of this channel and its empirical relevance during the Argentine currency crisis, trying to assess whether or not the dollar price of imported goods fell; if there were compositional changes in the set of imports; and if so, whether the replacement was in favor of cheaper varieties of lower quality.
\end{abstract}

Clasificación JEL: F10, F14, F31

Palabras clave: Devaluación, Traspaso a precios, Importaciones, Calidad, Argentina

\footnotetext{
*Este trabajo constituye la tesis de Maestría en Economía de la UNLP, realizada bajo la dirección de Irene Brambilla. A ella agradezco enormemente su valiosa guía y su apoyo. También agradezco el apoyo y las sugerencias de mis amigos del Centro de Estudios Distributivos Laborales y Sociales (CEDLAS) y de los participantes del seminario de tesis de Maestría. Los errores son de mi exclusiva responsabilidad.

${ }^{\dagger}$ Becario del CONICET - Facultad de Ciencias Económicas, Universidad Nacional de La Plata. E-mail: andres.cesar@econo.unlp.edu.ar
} 


\section{Índice}

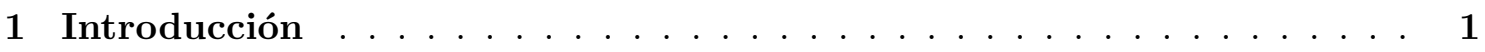

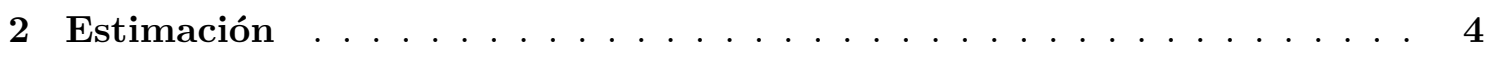

2.1 Datos utilizados . . . . . . . . . . . . . . . . . . 4

2.2 Estrategia de estimación . . . . . . . . . . . . . . . . . . 4

3 Resultados ........................... 8

3.1 Caída en los precios de importados . . . . . . . . . . . . . 8

3.2 Estimación de la demanda . . . . . . . . . . . . . . . . . . 9

3.3 Caída en la calidad de importados . . . . . . . . . . . . . . . . . . . . 11

3.4 Cambio en el set de importados . . . . . . . . . . . . . . . . . . 13

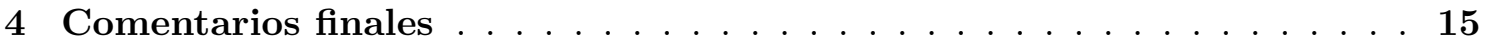

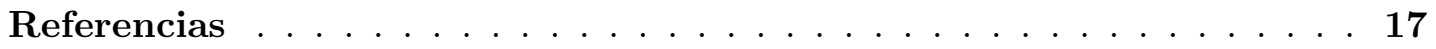

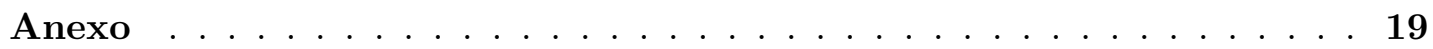




\section{Introducción}

El traspaso a precios de las variaciones en el tipo de cambio (pass-through) es y ha sido un tema muy estudiado en la literatura de economía internacional. En los modelos clásicos de libro de texto, los precios domésticos de los productos importados varían uno a uno con el tipo de cambio, y es válida internacionalmente la ley de precio único. Las primeras discusiones comenzaron a cuestionar la validez empírica de esta ley, y se fue gestando un consenso que avala la existencia de un traspaso incompleto desde las variaciones en el tipo de cambio hacia los precios internos (incomplete pass-through). Existe una amplia literatura dedicada a estudiar este tema. Los primeros trabajos para Estados Unidos encontraron que el pass-through se encontraba aproximadamente entre 50 y $70 \%$ (Kreinin (1977); Hooper y Mann (1989)). La mayoría de los trabajos que utilizan datos desagregados, encuentran variaciones en el pass-through para distintas industrias y/o países (Kravis y Lipsey (1978); Knetter (1989); Ohno (1988); Marston (1990); Campa y Goldberg (2006); Burstein y Gopinath (2013)).

Buena parte de la literatura de economía internacional comenzó a estudiar el fenómeno del pass-through de manera indirecta, al evaluar los efectos que tienen las variaciones del tipo de cambio sobre la balanza comercial ${ }^{3}$. Años más tarde, además de continuar analizando el fenómeno en sí, las investigaciones comenzaron a estudiar sus causas en profundidad, teniendo en cuenta los actores que están detrás de la fijación de precios, las heterogeneidades que se presentan entre las diferentes industrias, países, e incluso a lo largo del tiempo. A pesar de que las estimaciones de la magnitud del pass-through varían considerablemente entre países e industrias, todos los trabajos coinciden en que el traslado a precios es rezagado e incompleto (Woo (1984), Engel (1993), Parsley y Wei (2001), Campa y Goldberg (2005, 2006, 2010), entre muchos otros).

Otras discusiones más recientes que también tienen en cuenta el fenómeno del pass-through, tienen que ver con su influencia en la determinación de la política monetaria óptima y los regímenes de tipo de cambio óptimos en el contexto de modelos de Equilibrio General ${ }^{4}$. Estudiar este fenómeno en profundidad es de vital importancia para analizar el rol que debe jugar la política monetaria a la hora de contribuir a la estabilización macroeconómica, teniendo en cuenta diversos fenómenos globales; entre otros, la transmisión internacional de shocks y la volatilidad de los flujos de capital y de comercio.

$\mathrm{Al}$ intentar comprender las causas del pass-through incompleto, se plantan diversas explicaciones. El argumento más intuitivo, se relaciona con la presencia de bienes no transables (que no cuentan con insumos importados en su función de producción) en la canasta de consumo, cuyo precio en moneda local no se ve afectado frente a una devaluación de la moneda (la intuición de este resultado podemos encontrarla en un modelo teórico de com-

\footnotetext{
${ }^{3}$ Discusiones de este tipo quedan fuera del alcance de este trabajo. Sin embargo, pueden encontrarse referencias importantes en Branson (1972); Clark (1974); Magee (1974); Kreinin (1977); Kravis y Lipsey (1978); Krugman y Baldwin (1987); Helkie y Hooper (1987, 1989); entre muchos otros.

${ }^{4}$ Estos temas también se encuentran al margen de este trabajo. Sin embargo, pueden encontrarse referencias de importancia en Obstfeld (2000); Devereux y Engel (2000); Corsetti y Pesenti (2001); Devereux (2001); entre otros.
} 
petencia monopolística presentado en Bacchetta y van Wincoop (2003)). Más interesante y a la vez complejo resulta investigar este fenómeno en el conjunto de los bienes transables de la economía. Para el caso de los productos exportados, es de suponer que luego de la devaluación, los exportadores exigirán un precio mayor para vender los mismos productos que exportan en el mercado local. Campos (2010) analiza este fenómeno utilizando microdatos de las exportaciones de Brasil. Su trabajo incorpora los cambios que ocurren en el set firmas-productos de los mercados de exportación luego de las grandes devaluaciones (particularmente la del real en la década del noventa), como posible explicación adicional al fenómeno de pass-through incompleto. En el mismo sentido, el precio de los productos importados también aumenta luego de la devaluación, y curiosamente el pass-through también es incompleto para estos bienes. Aquí se encuentra el foco de interés de este trabajo. Más grande sea el pass-through mayor será la respuesta de los precios agregados de la economía y por tanto, mayor será el impacto de las devaluaciones de la moneda en la inflación. A su vez, este es el fenómeno que más ha estudiado la literatura, y se han presentado diversas respuestas tanto teóricas como empíricas.

Una primera explicación se relaciona con el poder de mercado de las firmas. Una de sus variantes se denomina "Doble marginalización", esta nace de suponer la existencia de competencia imperfecta en el sector de distribución (Hellerstein, 2004). La atención se centra en el rol que juegan los mayoristas y minoristas de la cadena de distribución (particularmente en la determinación estratégica del Mark-up). Luego de una devaluación, estos actores locales son quienes absorben la mayor parte del aumento de los costos. Esto se debe a que, en la búsqueda de mayores beneficios, estos agentes mantienen/disminuyen los precios para aumentar su participación en el mercado. Otras variantes del mismo razonamiento se encuentran en Dornbusch (1987), Bergin y Feenstra (2001), Atkeson y Burstein (2008). Muy relacionado con este argumento se encuentra el reciente trabajo de Amiti, Itskhoki y Konings (2012). Los autores se centran en el canal de los costos y en el ajuste del Mark-up, pero instalando en el eje de la discusión a las grandes firmas internacionales, que no solo son grandes importadoras sino que también son grandes exportadoras: ello genera un mecanismo de amortiguación cuando se enfrentan a variaciones en los tipos de cambio, lo que resulta clave para entender los bajos niveles de pass-through agregado.

La segunda explicación se basa en la existencia de costos de distribución domésticos. Existe evidencia que fundamenta la existencia de tales costos (transporte, almacenamiento, financiamiento, seguros, venta al por mayor y por menor, marketing) que agregan valor agregado local al precio final de los bienes importados, y por lo tanto reducen el impacto de cambios en los precios de dichos bienes sobre los precios internos (Burstein, Neves, y Rebelo (2003); Burstein, Eichembaum y Rebelo (2005); Corsetti y Dedola (2004)).

La tercera explicación se relaciona con las rigideces nominales de precios, muy vinculadas con la existencia de costos de ajuste (Devereux y Engel (2002); Gopinath y Itskhoki (2010)).

Nakamura y Zerom (2010) toman como base un modelo de oligopolio estructural con el objetivo de determinar la importancia relativa de los distintos factores mencionados hasta 
aquí. Utilizando microdatos de ventas y precios para la industria del café, encuentran que los costos locales son el principal factor explicativo del pass-through incompleto (78\%), seguido por el ajuste del Mark-up (20\%), y en último lugar los costos de ajuste (2\%). En este sentido, la tercera explicación presentada no sería tan relevante. Con un objetivo similar, Campa y Goldberg (2006) se basan en un modelo simple de dos países con rigidez salarial para realizar una contribución empírica interesante. El trabajo explora los canales de transmisión del tipo de cambio hacia los precios internos de distintos bienes de consumo y hacia el nivel de precios agregado de la economía, utilizando datos de 21 economías desarrolladas de la OECD. En promedio, el pass-through estimado es de 15\%. Por otro lado, los autores encuentran que los costos de distribución comprenden, en promedio, desde un $32 \%$ hasta un $50 \%$ del costo total de los bienes de consumo de los países estudiados. El otro hallazgo interesante es la evidencia a favor de la existencia del proceso de "Doble Marginalización".

La hipótesis de este trabajo, entendida como fenómeno de sustitución de variedades, entra como una explicación complementaria a las que ha propuesto la literatura. El estudio se concentra en los precios en aduana de los productos importados expresados en moneda extranjera. La devaluación genera una caída en el ingreso real y una consecuente menor disposición a pagar de parte de los consumidores. Ello traería consigo un cambio composicional en la canasta de importaciones, mediante la cual, para el mismo producto se sustituyen variedades de precio alto por variedades de precio bajo (y presumiblemente menor calidad ${ }^{5}$ ). Como consecuencia, esta sustitución produce una caída en los precios promedio de los productos importados, que actúa como un atenuante adicional de la suba de precios de estos mismos productos expresados en moneda local.

Un antecedente relacionado con esta hipótesis, aparece como un resultado complementario en Burstein, Eichembaum y Rebelo (2005). Los autores se proponen explicar los factores que están detrás de la baja tasa de inflación luego de las grandes devaluaciones, encontrando que el contenido doméstico del costo de los bienes importados es significativo a la hora de explicar el pass-through incompleto. En su trabajo, realizan una serie de ejercicios para simular la inflación real del período, obteniendo resultados bastante acertados, pero no idénticos. Las causas del "desvío" son atribuídas a dos factores: el primero se asocia a un error de especificación originado por suponer que el sector de distribución es perfectamente competitivo; el segundo factor se vincula directamente con este trabajo y se denomina "fly from quality", y es definido como la sustitución hacia bienes de menor calidad producidos localmente que llevan a cabo los consumidores en la etapa posterior a las grandes devaluaciones. La hipótesis de este trabajo también encuentra soporte en dos trabajos que estudian el período de crisis argentina de 2001-2002. Gopinath y Neiman (2014) estudian el mecanismo de ajuste comercial que ocurrió luego de la devaluación de 2002, y encuentran que el margen extensivo (entrada y salida de firmas) jugó un rol menor, siendo el factor de ajuste principal el margen sub-extensivo (o intensivo) al interior de las

\footnotetext{
${ }^{5}$ En primera instancia, esta es entendida como fenómeno puramente subjetivo, y se vincula directamente con las elecciones que hacen los consumidores. Las variantes metodológicas utilizadas para estimar calidad siguen la discusión presentada en Khandelwal (2010).
} 
firmas: quienes ajustaron el mix de insumos importados. Encuentran que este fenómeno, sumado a otros factores, ha tenido un impacto negativo en la productividad de las firmas. Por su parte, McKenzie y Schargrodsky (2011) realizan un estudio de la crisis argentina basado en encuestas de consumo de los hogares argentinos, documentando la existencia de sustitución en el consumo doméstico hacia productos de menor precio y menor calidad, como un mecanismo adicional para suavizar la crisis económica.

El resto del trabajo se estructura como sigue. En la segunda sección, se mencionan las fuentes de datos y se explica la estrategia de estimación empleada a lo largo del trabajo. En la tercera sección se discuten los resultados obtenidos. La cuarta sección concluye.

\section{Estimación}

Se realizan diversas estimaciones econométricas donde se busca comparar los precios y la composición de la canasta de importaciones antes y después de la crisis 2001-2002, con el objetivo de encontrar racionalidad estadística y econométrica de las hipótesis propuestas.

\subsection{Datos utilizados}

Los datos provienen de dos fuentes: (i) INDEC, que recopila datos del valor y volumen de importaciones (por país de origen y a un nivel de desagregación de 8 dígitos), y los mismos están disponibles a través del Departamento de pedidos especiales. (ii) COMTRADE, de la División de Estadística de Naciones Unidas, la cual recopila datos bilaterales de comercio internacional con un nivel de desagregación de 6 dígitos. Estos últimos se utilizan para construir precios de referencia mundiales que sirven como instrumentos para los precios de aduana de las importaciones argentinas. Los base de datos es de acceso público, ingresando a la sección database (Comtrade, UNCTAD).

Con los datos disponibles, se construye un panel de importaciones argentinas desagregadas por tipo de producto y país de origen, con el máximo nivel de desagregación que es posible encontrar en nuestro país (8 dígitos del Sistema Armonizado). Se tiene en cuenta el período de seis años que circunscribió a la crisis argentina (1999-2004). Más allá de concentrarnos en un panel corto, la inferencia arrojara estimadores consistentes pues se cuenta con un número grande de observaciones para cada año.

\subsection{Estrategia de estimación}

El primer set de regresiones es más bien descriptivo de los fenómenos que se intentan comprender, y se utiliza el método de Mínimos Cuadrados Ordinarios (MCO). El objetivo es determinar qué ocurrió con el precio de los productos y variedades importadas en los años previos y posteriores a la devaluación del 2002, mediante la siguiente ecuación de regresión: 


$$
\ln p_{j h t}=\beta \text { Post }+E F_{j h}+\varepsilon_{j h t}
$$

La variable dependiente es el precio de las variedades importadas, medido como el logaritmo natural del valor unitario ${ }^{6}$ en dólares de las importaciones argentinas de la variedad $h j$. Una variedad se define como una combinación $\operatorname{producto}(h)$-país $(j)$. La variable dummy Post, toma el valor cero para los años previos a la devaluación, y el valor uno para los años posteriores (2002 en adelante ${ }^{7}$ ). Esta es la variable de interés, pues el coeficiente $\beta$ intenta capturar el efecto diferencial en los precios promedio en los años posteriores a la crisis cambiaria. Se incorporan Efectos Fijos (EF) por variedad; y $\varepsilon_{j h t}$ son los errores de estimación. En una segunda especificación se reemplaza a la variable Post por dummies anuales que permitirán comparar los precios promedio de cada año respecto al año base (1999).

Tanto estas estimaciones como las sucesivas, se realizan a nivel variedad y a nivel producto, con errores estándar calculados por clusters a nivel variedad (o producto). El precio de un producto es calculado como el precio promedio de todas las variedades que lo componen, ponderado a cada una por su participación en el total importado dentro de esa categoría de producto. Además, las estimaciones se realizan para un período de 4 años (2000-2003) y para uno de 6 años (1999-2004).

Dado que es natural suponer que precios más altos son una señal de mayor calidad, los precios pueden pensarse como una medida "cruda" de la calidad. Sin embargo, es perfectamente posible que esto no suceda. Los datos disponibles no permiten inferir calidad a partir de características observables de los productos importados, por lo tanto es necesario contar con algún método que permita estimar "calidad". Para ello, se adopta una forma paramétrica que sigue la metodología empleada en Khandelwal $(2010)^{8}$. La estimación parte de suponer que el consumidor debe elegir una variedad entre todas las alternativas disponibles. Los consumidores tienen una función de utilidad definida sobre productos vertical y horizontalmente diferenciados, que sigue un Logit Multinomial. La utilidad que el consumidor $i$ deriva de elegir el producto $h$ que proviene del país $j$ es:

$$
U_{i j h t}=\theta_{i j h t}-\alpha p_{i j h t}+\varepsilon_{i j h t}
$$

Donde $\theta$ es la calidad subjetiva de la variedad, $p$ es su precio, $\alpha$ es la disposición a pagar del consumidor y $\varepsilon$ es un shock idiosincrático. El shock puede ser independiente e idénticamente distribuido o estar correlacionado entre productos, lo que da origen a un Nested Logit. Al agregar las decisiones de los individuos (aleatorias a nivel individual, pero determinísticas a nivel agregado por la Ley de los Grandes Números) se obtiene la función

\footnotetext{
${ }^{6} \mathrm{El}$ precio o valor unitario de cada producto se calcula como el valor CIF dividido las cantidades importadas. ${ }^{7}$ Durante los primeros meses de 2002 la moneda Argentina se depreció alrededor de $300 \%$.

${ }^{8} \mathrm{El}$ autor infiere calidad a partir de diferencias en precios y market shares, y encuentra que los precios promedio son una medida imprecisa de la calidad en productos que presentan "Quality ladders" más cortas, debido al componente de diferenciación horizontal que hace que coexistan bienes con diferentes calidades y precios similares. En cambio, los precios resultan ser medidas más precisas de la calidad en aquellos productos que presentan "Quality ladders" más largas.
} 
de demanda agregada de cada variedad:

$$
\ln s_{j h t}-\ln s_{0 t}=\theta_{j h t}-\alpha p_{j h t}
$$

Expresada como participación de cada variedad en el consumo total de importaciones de la industria a la que pertenece $\left(s_{j h t}\right)$, normalizada con respecto a una alternativa doméstica "0", que representa un sustituto cercano de los productos importados en cada industria 9 . Para calcular esta medida, se observan las cantidades totales importadas por cada industria para cada año y al máximo valor se le suma un $x \%$ (supuesta participación de la alternativa doméstica). Se supone que este valor refleja el tamaño total del mercado $(M)$. A partir de este valor es posible calcular la participación en cada una de las industrias. La intuición detrás de este supuesto es muy simple: en aquellas industrias que posean mayores cantidades importadas, la participación de la alternativa doméstica será menor. Se tienen en cuenta las 13 industrias de bienes manufacturados, agregando grupos de categorías de productos a 2 dígitos: Alimentos manufacturados; Textiles; Químicos; Maquinaria y maquinaria eléctrica; entre otras ${ }^{10}$.

La intuición detrás de la demanda agregada es clara: si dos variedades tienen el mismo precio, será mayor la participación de la variedad que tenga mayor calidad. Y viceversa, si dos variedades tienen la misma calidad, será mayor la participación de la variedad que tenga un precio menor.

A partir de la función de demanda agregada, se construye la ecuación de regresión:

$$
\ln \left(s_{j h t}\right)-\ln \left(s_{0 t}\right)=-\alpha_{1} \ln \left(p_{j h t}\right)-\alpha_{2} \ln \left(p_{j h t}\right) \text { Post }+\beta \ln \left(n s_{j h t}\right)+\theta_{j h}+\theta_{t j}+\theta_{t h}+\varepsilon_{j h t}
$$

La variable dependiente es el logaritmo (normalizado) de la participación de cada variedad en el consumo total de importaciones de la industria a la que pertenece. Naturalmente, un mismo producto es importado de distintos países: la variable $n s_{j h t}$ es la participación que tiene el país de origen $j$ en el total importado del producto $h$. Este término se incorpora para permitir cierta correlación en la estructura de preferencias de los consumidores, y es el que da origen al mencionado Nested Logit. Los coeficientes estimados $\alpha_{1}$ y $\alpha_{2}$ capturan la disposición a pagar de los consumidores. Se permite que esta sea diferente antes y después de la crisis, pues este es el motor de nuestro argumento. De igual manera, también se permitirá que la disposición a pagar varíe por industrias, reconociendo que el trade-off entre precio y calidad puede no ser el mismo para maquinarias que para productos textiles, por ejemplo. La calidad estimada $\hat{\theta}_{j h t}$ adopta una forma paramétrica, y se construye a partir de los efectos fijos y del error de estimación:

\footnotetext{
${ }^{9}$ Para construir esta medida, Khandelwal (2010) utiliza datos de "import penetration" para las diferentes industrias. Dado que en los datos disponibles no se cuenta con dicha información, es necesario realizar algún tipo de supuesto para calcular la participación de la variedad doméstica en cada industria. El supuesto es arbitrario y no afecta los resultados de las estimaciones realizadas.

${ }^{10}$ La tabla A1 del anexo enumera las industrias contempladas a lo largo de las estimaciones.
} 


$$
\hat{\theta}_{j h t}=\hat{\theta}_{j h}+\hat{\theta}_{t j}+\hat{\theta}_{t h}+\hat{\varepsilon}_{j h t}
$$

Donde $\left(\hat{\theta}_{j h}\right)$ representa un efecto fijo a nivel variedad, $\left(\hat{\theta}_{t j}\right)$ un efecto fijo a nivel añopaís, $\left(\hat{\theta}_{t h}\right)$ un efecto fijo a nivel año-producto y $\hat{\varepsilon}_{j h t}$ un componente no observado que varía por año-variedad (error de estimación del modelo). La intuición es que la suma de estos términos refleja la valoración por la variedad $j h$ que es común a través de todos los consumidores. Esta medida de calidad no puede separar la tecnología que tiene incorporada una variedad, de la valoración subjetiva por la calidad que tienen los consumidores. Este es el supuesto de identificación de nuestro modelo. Como prueba de robustez, se estimarán seis medidas de calidad alternativas mediante distintas combinaciones de efectos fijos.

La primera estimación se realiza por el método de MCO. Sin embargo, dado que en un sistema de oferta y demanda precios y cantidades se determinan simultáneamente, el término de error del modelo está potencialmente correlacionado con los precios unitarios (y con el componente no observado de la calidad). Es por ello que se realiza una segunda estimación por el método de Variables Instrumentales (VI). Es necesario contar con instrumentos que generen variaciones exógenas en la oferta que permitan identificar la demanda. El primer instrumento (tradicional en la literatura) para la variable $p_{j h t}$ son los costos de importación, definidos como la diferencia entre el precio CIF y el precio FOB. El segundo instrumento son los precios de referencia mundiales, construidos a partir de la base de datos de COMTRADE. Estos reflejan el precio promedio de cada producto (a 6 dígitos) entre los diferentes destinos hacia los cuales un país exporta dicho producto (y estarían reflejando shocks de costos en los países de origen). Por otro lado, $n s_{j h t}$ es otra variable potencialmente endógena, de modo que también se instrumenta a partir de una proxy (a nivel variedad) que es la combinación (suma) entre el número de variedades exportadas por cada país y la cantidad de países que exporta cada producto.

A partir de las distintas especificaciones, se obtendrán valores estimados para la disposición a pagar $(\hat{\alpha})$, y para la calidad de cada variedad $\left(\hat{\theta}_{j h t}\right)$. Ello permitirá establecer si hay una caída en la disposición a pagar, si hay una caída en la calidad de las variedades importadas, y si efectivamente existe sustitución hacia variedades de menor calidad y de menor precio. Para evaluar si la calidad efectivamente cayó estimamos la siguiente ecuación de regresión:

$$
\ln \left(\hat{\theta}_{j h t}\right)=\beta \text { Post }+E F_{j h}+\varepsilon_{j h t}
$$

El coeficiente $\beta$ intenta capturar el efecto diferencial en la calidad promedio en los años posteriores a la crisis cambiaria. Se incorporan Efectos Fijos $(\mathrm{EF})$ por variedad; y $\varepsilon_{j h t}$ son los errores de estimación. En una segunda especificación se reemplaza a la variable Post por dummies anuales que permitirán comparar la calidad promedio de cada año respecto al año base (1999).

A continuación, se intentará evaluar si existe alguna correlación entre la caída en la calidad de una variedad y el ingreso del país desde el cual proviene. Para ello, se divide a los países 
de origen en función de su ingreso per cápita, en cinco categorías: ingresos muy bajos (hasta el percentil 10), ingresos bajos (entre el $p 10$ y el $p 25$ ), ingresos medios (entre el $p 25 \mathrm{y}$ el $p 75$ ), ingresos altos (entre el $p 75$ y el $p 90$ ) e ingresos muy altos (desde el $p 90$ ). De igual forma, se estima la última ecuación de regresión pero interactuando ahora a la variable Post con la variable que tiene en cuenta de qué grupo de país proviene cada variedad.

Por último, para testear la hipótesis de sustitución de variedades, se estiman variantes de la siguiente ecuación de regresión:

$$
s h_{j h t}=\alpha_{1} \hat{\theta}_{j h t}+\alpha_{2} \text { Post }+\beta \hat{\theta}_{j h t} \text { Post }+E F_{j h}+\varepsilon_{j h t}
$$

La variable explicada es la participación de cada variedad en el total importado dentro de la categoría de producto a la que pertenece. En una segunda especificación, pasa a ser la participación de cada producto en el total importado dentro de la industria. El coeficiente de interés es $\beta$, que acompaña a la interacción entre la variable Post y la calidad estimada. De este modo, un coeficiente negativo para $\beta$ estaría indicando que las variedades que tienen mayor (menor) calidad pierden (ganan) participación en la canasta de consumo durante el período de la crisis cambiaria. Es decir, existe sustitución hacia variedades de menor calidad. Similarmente, se estima la ecuación de regresión pero reemplazando la calidad estimada por el precio, para evaluar si existe sustitución hacia variedades de menor precio.

\section{Resultados}

\subsection{Caída en los precios de importados}

Con el primer set de regresiones se intenta mostrar que los precios en aduana de las variedades y productos importados disminuyen en los años posteriores a la crisis cambiaria. La tabla 1 presenta los coeficientes ( $\beta$ ) estimados que acompañan a la variable Post, en las columnas 1, 2, 4 y 5; y los coeficientes estimados que acompañan a las dummies anuales, en las columnas 3 y 6 . Las figuras 1 a 4 en el anexo presentan histogramas del cambio en el logaritmo natural de los precios, que siguen la misma línea que los resultados que se comentan a continuación. Para la elaboración de los histogramas se eligen a modo ilustrativo los años 2000 y 2002. 
Tabla 1. Cambio en precios de importados a nivel variedad y producto

\begin{tabular}{|c|c|c|c|c|c|c|}
\hline & \multicolumn{3}{|c|}{ Ln (Precio a nivel Variedad) } & \multicolumn{3}{|c|}{ Ln (Precio a nivel Producto) } \\
\hline & (1) & $(2)$ & $(3)$ & (4) & $(5)$ & (6) \\
\hline Post & $\begin{array}{c}-0.051^{*} \\
(0.03)\end{array}$ & $\begin{array}{c}-0.104^{* * *} \\
(0.02)\end{array}$ & & $\begin{array}{l}-0.038 \\
(0.033)\end{array}$ & $\begin{array}{c}-0.078^{* * *} \\
(0.029)\end{array}$ & \\
\hline Año 2000 & & & $\begin{array}{c}0.013 \\
(0.024)\end{array}$ & & & $\begin{array}{c}0.021 \\
(0.028)\end{array}$ \\
\hline Año 2001 & & & $\begin{array}{l}-0.006 \\
(0.028)\end{array}$ & & & $\begin{array}{l}-0.008 \\
(0.026)\end{array}$ \\
\hline Año 2002 & & & $\begin{array}{c}-0.117^{* * *} \\
(0.035)\end{array}$ & & & $\begin{array}{c}-0.087^{* *} \\
(0.044)\end{array}$ \\
\hline Año 2003 & & & $\begin{array}{c}-0.099 * * * \\
(0.038)\end{array}$ & & & $\begin{array}{c}-0.082^{* *} \\
(0.04)\end{array}$ \\
\hline Año 2004 & & & $\begin{array}{c}0.031 \\
(0.048)\end{array}$ & & & $\begin{array}{c}0.039 \\
(0.044)\end{array}$ \\
\hline Observaciones & 297,244 & 182,799 & 297,244 & 38,220 & 24,807 & 38,220 \\
\hline R-cuadrado & 0.011 & 0.078 & 0.062 & 0.007 & 0.042 & 0.050 \\
\hline Variedades & 71,061 & 58,291 & 71,061 & & & \\
\hline Productos & & & & 7,425 & 6,988 & 7,425 \\
\hline EF variedad & $\mathrm{Si}$ & $\mathrm{Si}$ & $\mathrm{Si}$ & & & \\
\hline EF producto & & & & $\mathrm{Si}$ & $\mathrm{Si}$ & $\mathrm{Si}$ \\
\hline
\end{tabular}

Columnas (1), (3), (4), (6): período 1999 a 2004.

Columnas (2), (5): período 2000 a 2003.

El precio promedio de las variedades importadas (en dólares) en los tres años posteriores a la crisis cambiaria resulta ser un $5 \%$ menor que en los tres años previos. Cuando reducimos el período de comparación a dos años, la caída promedio resulta ser mayor a $10 \%$. Ello es consecuencia de que la mayor parte de la caída en precios se produce en los años 2002 (poco menos de $12 \%$ respecto a 1999) y 2003 (poco menos de $10 \%$ ). Como se dijo, para calcular la variación en el precio de los productos, se pondera a las variedades por su participación importadora dentro de la categoría de producto a la que pertenecen. Los resultados obtenidos presentan la misma tendencia.

\subsection{Estimación de la demanda}

Se pasa ahora a analizar los parámetros estimados a partir de la ecuación de la demanda. La tabla 2 presenta los coeficientes de la disposición a pagar de los consumidores $(\alpha)$, estimados por MCO (panel A) y por VI (panel B). En la primera especificación se estima la disposición a pagar que es común a todos los consumidores, mientras que en las especificaciones 2 a 5 se permite que la disposición a pagar varíe por industrias. En este último caso, se reporta el promedio de los coeficientes estimados. Las tablas A2 y A3 del Anexo presentan la información detallada de cada una de las estimaciones. Como se dijo, 
a partir de las distintas especificaciones presentadas será posible obtener diversas medidas de calidad que se utilizarán en las estimaciones que siguen.

Tabla 2. Estimación de parámetros de la demanda

\begin{tabular}{|c|c|c|c|c|c|c|}
\hline & \multicolumn{6}{|c|}{ Log Share de la Variedad Normalizado } \\
\hline & (1) & $(2)$ & $(3)$ & (4) & $(5)$ & $(6)$ \\
\hline \multicolumn{7}{|l|}{ Panel A: MCO } \\
\hline \multirow[t]{2}{*}{ Precio } & $-0.121^{* * *}$ & & & & & \\
\hline & 0.003 & & & & & \\
\hline Coef. Precio Promedio & & -0.143 & -0.128 & -0.143 & -0.773 & -0.128 \\
\hline Significativos al $1 \%$ & & $12 / 13$ & $13 / 13$ & $12 / 13$ & $13 / 13$ & $13 / 13$ \\
\hline Precio * Post & $\begin{array}{c}-0.012^{* * *} \\
(0.002)\end{array}$ & $\begin{array}{c}-0.012^{* * *} \\
(0.002)\end{array}$ & $\begin{array}{c}-0.023^{* * *} \\
(0.002)\end{array}$ & $\begin{array}{c}-0.019 * * * \\
(0.002)\end{array}$ & $\begin{array}{c}-0.028^{* * *} \\
(0.005)\end{array}$ & $\begin{array}{c}-0.025^{* * *} \\
(0.002)\end{array}$ \\
\hline Sigma & $\begin{array}{c}0.881^{* * *} \\
(0.002)\end{array}$ & $\begin{array}{c}0.879^{* * *} \\
(0.002)\end{array}$ & $\begin{array}{c}0.882^{* * *} \\
(0.002)\end{array}$ & $\begin{array}{c}0.879 * * * \\
(0.002)\end{array}$ & $\begin{array}{c}0.595^{* * *} \\
(0.016)\end{array}$ & $\begin{array}{c}0.881^{* * *} \\
(0.002)\end{array}$ \\
\hline Observaciones & 297,244 & 297,244 & 297,244 & 297,244 & 325,449 & 297,244 \\
\hline Variedades & 71,061 & 71,061 & 71,061 & 71,061 & 71,061 & 71,061 \\
\hline \multicolumn{7}{|l|}{ Panel B: VI } \\
\hline \multirow[t]{2}{*}{ Precio } & $-0.370^{* * *}$ & & & & & \\
\hline & 0.041 & & & & & \\
\hline Coef. Precio Promedio & & -0.639 & -0.810 & -0.750 & -1.122 & -1.002 \\
\hline Significativos al $1 \%$ & & $13 / 13$ & $13 / 13$ & $13 / 13$ & $13 / 13$ & $13 / 13$ \\
\hline Precio $*$ Post & $\begin{array}{c}-0.022^{* * *} \\
(0.002)\end{array}$ & $\begin{array}{c}-0.019 * * * \\
(0.002)\end{array}$ & $\begin{array}{c}-0.019 * * * \\
(0.002)\end{array}$ & $\begin{array}{c}-0.026^{* * *} \\
(0.002)\end{array}$ & $\begin{array}{c}-0.030^{* * *} \\
(0.008)\end{array}$ & $\begin{array}{c}-0.017^{* * *} \\
(0.004)\end{array}$ \\
\hline Sigma & $\begin{array}{c}0.755^{* * *} \\
(0.032)\end{array}$ & $\begin{array}{c}0.740^{* * *} \\
(0.034)\end{array}$ & $\begin{array}{c}0.653^{* * *} \\
(0.036)\end{array}$ & $\begin{array}{c}0.682^{* * * *} \\
(0.042)\end{array}$ & $\begin{array}{c}0.561^{* * *} \\
(0.051)\end{array}$ & $\begin{array}{c}0.556^{* * *} \\
(0.058)\end{array}$ \\
\hline Observaciones & 293,680 & 293,680 & 293,680 & 293,680 & 321,645 & 293,680 \\
\hline Variedades & 70,294 & 70,294 & 70,294 & 70,294 & & 70,294 \\
\hline EF año & $\mathrm{Si}$ & $\mathrm{Si}$ & No & No & No & No \\
\hline EF año-industria & No & No & $\mathrm{Si}$ & No & $\mathrm{Si}$ & $\mathrm{Si}$ \\
\hline EF año-país & No & No & No & $\mathrm{Si}$ & $\mathrm{Si}$ & $\mathrm{Si}$ \\
\hline EF producto-país & $\mathrm{Si}$ & $\mathrm{Si}$ & $\mathrm{Si}$ & $\mathrm{Si}$ & No & $\mathrm{Si}$ \\
\hline
\end{tabular}

Todos los coeficientes estimados de la disposición a pagar arrojan signos consistentes (negativos) con una ecuación de demanda. Los resultados siguen la línea de la primera hipótesis -y motor del argumento-: la disposición a pagar cae en los años posteriores a la crisis cambiaria. Al estimar por el método de VI, los coeficientes se mueven en la dirección esperada: su magnitud aumenta de manera considerable. La caída en la disposición a pagar es un resultado robusto a través de todas las especificaciones presentadas. Siguiendo con el argumento, la reducción en la disposición a pagar podría generar un cambio composicional en el set de importaciones. Esto es lo que se intenta estudiar a continuación. 


\subsection{Caída en la calidad de importados}

A partir de las estimaciones del sistema de demanda, se dispone de doce medidas de calidad alternativas que permitirán establecer qué sucede con la calidad de las variedades y productos importados en los años posteriores a la crisis cambiaria. Para ello, se estima la ecuación de regresión comentada en la sección previa. La tabla 3 presenta los resultados a nivel variedad. La tabla A3 del anexo, presenta los mismos resultados a nivel producto. Los paneles A y B presentan el coeficiente estimado para la variación promedio de la calidad estimada de las variedades para todo el período considerado. El panel C reporta los coeficientes estimados para las dummies anuales (tomando el año 1999 como base).

Tabla 3. Cambio en la calidad a nivel variedad

\begin{tabular}{|c|c|c|c|c|c|c|}
\hline & \multicolumn{6}{|c|}{ Calidad } \\
\hline & (1) & $(2)$ & $(3)$ & $(4)$ & $(5)$ & $(6)$ \\
\hline \multicolumn{7}{|l|}{ Panel A: MCO } \\
\hline Post & $\begin{array}{c}-0.745^{* * *} \\
(0.004)\end{array}$ & $\begin{array}{c}-0.748^{* * *} \\
(0.004)\end{array}$ & $\begin{array}{c}-0.716^{* * *} \\
(0.004)\end{array}$ & $\begin{array}{c}-0.729^{* * *} \\
(0.004)\end{array}$ & $\begin{array}{c}-0.757^{* * *} \\
(0.005)\end{array}$ & $\begin{array}{c}-0.710^{* * *} \\
(0.004)\end{array}$ \\
\hline Observaciones & 297,244 & 297,244 & 297,244 & 297,244 & 297,244 & 297,244 \\
\hline R-cuadrado & 0.181 & 0.180 & 0.168 & 0.173 & 0.141 & 0.166 \\
\hline Variedades & 71,061 & 71,061 & 71,061 & 71,061 & 71,061 & 71,061 \\
\hline \multicolumn{7}{|l|}{ Panel B: VI } \\
\hline Post & $\begin{array}{c}-0.735^{* * *} \\
(0.004)\end{array}$ & $\begin{array}{c}-0.751^{* * *} \\
(0.004)\end{array}$ & $\begin{array}{c}-0.768^{* * *} \\
(0.005)\end{array}$ & $\begin{array}{c}-0.741^{* * *} \\
(0.005)\end{array}$ & $\begin{array}{c}-0.754^{* * *} \\
(0.005)\end{array}$ & $\begin{array}{c}-0.793^{* * *} \\
(0.005)\end{array}$ \\
\hline Observaciones & 293,680 & 293,680 & 293,680 & 293,680 & 293,680 & 293,680 \\
\hline R-cuadrado & 0.169 & 0.171 & 0.162 & 0.157 & 0.134 & 0.149 \\
\hline Variedades & 70,294 & 70,294 & 70,294 & 70,294 & 70,294 & 70,294 \\
\hline \multicolumn{7}{|c|}{ Panel C: VI. Por año } \\
\hline Año 2000 & $\begin{array}{c}0.067^{* * *} \\
(0.004)\end{array}$ & $\begin{array}{c}0.097^{* * *} \\
(0.004)\end{array}$ & $\begin{array}{c}0.090^{* * *} \\
(0.004)\end{array}$ & $\begin{array}{c}0.092^{* * *} \\
(0.004)\end{array}$ & $\begin{array}{c}0.079^{* * *} \\
(0.005)\end{array}$ & $\begin{array}{c}0.083^{* * *} \\
(0.005)\end{array}$ \\
\hline Año 2001 & $\begin{array}{c}-0.298^{* * *} \\
(0.004)\end{array}$ & $\begin{array}{c}-0.263^{* * *} \\
(0.004)\end{array}$ & $\begin{array}{c}-0.274^{* * *} \\
0.004\end{array}$ & $\begin{array}{c}-0.271^{* * *} \\
(0.004)\end{array}$ & $\begin{array}{c}-0.291^{* * *} \\
(0.005)\end{array}$ & $\begin{array}{c}-0.287^{* * * *} \\
(0.005)\end{array}$ \\
\hline Año 2002 & $\begin{array}{c}-1.622^{* * *} \\
(0.006)\end{array}$ & $\begin{array}{c}-1.606^{* * *} \\
(0.006)\end{array}$ & $\begin{array}{c}-1.615^{* * *} \\
(0.006)\end{array}$ & $\begin{array}{c}-1.591^{* * *} \\
(0.006)\end{array}$ & $\begin{array}{c}-1.595^{* * *} \\
(0.007)\end{array}$ & $\begin{array}{c}-1.630^{* * *} \\
(0.007)\end{array}$ \\
\hline Año 2003 & $\begin{array}{c}-0.789^{* * *} \\
(0.006)\end{array}$ & $\begin{array}{c}-0.780^{* * *} \\
(0.006)\end{array}$ & $\begin{array}{c}-0.807^{* * *} \\
(0.006)\end{array}$ & $\begin{array}{c}-0.776^{* * *} \\
(0.006)\end{array}$ & $\begin{array}{c}-0.806^{* * *} \\
(0.007)\end{array}$ & $\begin{array}{c}-0.842^{* * *} \\
(0.007)\end{array}$ \\
\hline Año 2004 & $\begin{array}{c}-0.136 * * * \\
(0.006)\end{array}$ & $\begin{array}{c}-0.141^{* * *} \\
(0.006)\end{array}$ & $\begin{array}{c}-0.174^{* * *} \\
(0.006)\end{array}$ & $\begin{array}{c}-0.141^{* * *} \\
(0.006)\end{array}$ & $\begin{array}{c}-0.177^{* * *} \\
(0.007)\end{array}$ & $\begin{array}{c}-0.216^{* * *} \\
(0.007)\end{array}$ \\
\hline Observaciones & 293,680 & 293,680 & 293,680 & 293,680 & 293,680 & 293,680 \\
\hline R-cuadrado & 0.418 & 0.405 & 0.368 & 0.376 & 0.308 & 0.323 \\
\hline Variedades & 70,294 & 70,294 & 70,294 & 70,294 & 70,294 & 70,294 \\
\hline
\end{tabular}

Las doce medidas de calidad estimadas dan cuenta de una reducción en la calidad de las variedades y productos importados en los años posteriores a la crisis cambiaria. En el 
panel $\mathrm{C}$ se advierte que la calidad promedio de las variedades comienza a descender ya en el año 2001, pero la caída de mayor magnitud se produce en el año de la devaluación, y continúa en los dos años siguientes (disminuyendo gradualmente). Estos resultados se replican a nivel producto (ver tabla A4 en el anexo), y son robustos frente a todas las especificaciones de calidad utilizadas.

La tabla 4 presenta los resultados de las estimaciones que tienen en cuenta el ingreso del país de origen. Como antes, el panel A considera las medidas de calidad construidas a partir de las estimaciones por MCO, mientras que el panel B las construidas a partir de la estimación por VI.

Tabla 4. Cambio en la calidad y país de origen

\begin{tabular}{|c|c|c|c|c|c|c|}
\hline & \multicolumn{6}{|c|}{ Calidad } \\
\hline & (1) & $(2)$ & $(3)$ & $(4)$ & $(5)$ & $(6)$ \\
\hline \multicolumn{7}{|l|}{ Panel A: MCO } \\
\hline Post $*$ Ingresos muy bajos & $\begin{array}{c}-1.114^{* * *} \\
(0.013)\end{array}$ & $\begin{array}{c}-1.105^{* * *} \\
(0.013)\end{array}$ & $\begin{array}{c}-1.085^{* * *} \\
(0.013)\end{array}$ & $\begin{array}{c}-1.093^{* * *} \\
(0.013)\end{array}$ & $\begin{array}{c}-1.069 * * * \\
(0.015)\end{array}$ & $\begin{array}{c}-1.081^{* * *} \\
(0.013)\end{array}$ \\
\hline Post $*$ Ingresos bajos & $\begin{array}{c}-0.640^{* * *} \\
(0.011)\end{array}$ & $\begin{array}{c}-0.624^{* * *} \\
(0.011)\end{array}$ & $\begin{array}{c}-0.604^{* * *} \\
(0.011)\end{array}$ & $\begin{array}{c}-0.612^{* * *} \\
(0.011)\end{array}$ & $\begin{array}{c}-0.540 * * * \\
(0.013)\end{array}$ & $\begin{array}{c}-0.600 * * * \\
(0.011)\end{array}$ \\
\hline Post $*$ Ingresos medios & $\begin{array}{c}-0.826^{* * *} \\
(0.006)\end{array}$ & $\begin{array}{c}-0.832^{* * *} \\
(0.006)\end{array}$ & $\begin{array}{c}-0.798^{* * *} \\
(0.006)\end{array}$ & $\begin{array}{c}-0.813^{* * *} \\
(0.006)\end{array}$ & $\begin{array}{c}-0.865^{* * *} \\
(0.007)\end{array}$ & $\begin{array}{c}-0.793^{* * *} \\
(0.006)\end{array}$ \\
\hline Post $*$ Ingresos altos & $\begin{array}{c}-0.746^{* * *} \\
(0.009)\end{array}$ & $\begin{array}{c}-0.755^{* * *} \\
(0.009)\end{array}$ & $\begin{array}{c}-0.717^{* * *} \\
(0.009)\end{array}$ & $\begin{array}{c}-0.733^{* * *} \\
(0.009)\end{array}$ & $\begin{array}{c}-0.779 * * * \\
(0.01)\end{array}$ & $\begin{array}{c}-0.711^{* * *} \\
(0.009)\end{array}$ \\
\hline Post * Ingresos muy altos & $\begin{array}{c}-0.358^{* * *} \\
(0.01)\end{array}$ & $\begin{array}{c}-0.366^{* * *} \\
(0.01)\end{array}$ & $\begin{array}{c}-0.326^{* * *} \\
(0.01)\end{array}$ & $\begin{array}{c}-0.343^{* * *} \\
(0.01)\end{array}$ & $\begin{array}{c}-0.411^{* * *} \\
(0.012)\end{array}$ & $\begin{array}{c}-0.319^{* * *} \\
(0.01)\end{array}$ \\
\hline Observaciones & 297,244 & 297,244 & 297,244 & 297,244 & 297,244 & 297,244 \\
\hline R-cuadrado & 0.196 & 0.195 & 0.183 & 0.187 & 0.152 & 0.180 \\
\hline Variedades & 71,061 & 71,061 & 71,061 & 71,061 & 71,061 & 71,061 \\
\hline \multicolumn{7}{|l|}{ Panel B: VI } \\
\hline Post $*$ Ingresos muy bajos & $\begin{array}{c}-1.114^{* * *} \\
(0.014)\end{array}$ & $\begin{array}{c}-1.090 * * * \\
(0.014)\end{array}$ & $\begin{array}{c}-1.087^{* * *} \\
(0.015)\end{array}$ & $\begin{array}{c}-1.074^{* * *} \\
(0.014)\end{array}$ & $\begin{array}{c}-1.066^{* * *} \\
(0.016)\end{array}$ & $\begin{array}{c}-1.088^{* * *} \\
(0.016)\end{array}$ \\
\hline Post $*$ Ingresos bajos & $\begin{array}{c}-0.626^{* * *} \\
(0.011)\end{array}$ & $\begin{array}{c}-0.580 * * * \\
(0.011)\end{array}$ & $\begin{array}{c}-0.562^{* * *} \\
(0.012)\end{array}$ & $\begin{array}{c}-0.553^{* * *} \\
(0.012)\end{array}$ & $\begin{array}{c}-0.527^{* * *} \\
(0.013)\end{array}$ & $\begin{array}{c}-0.545^{* * *} \\
(0.013)\end{array}$ \\
\hline Post $*$ Ingresos medios & $\begin{array}{c}-0.818^{* * *} \\
(0.006)\end{array}$ & $\begin{array}{c}-0.847^{* * *} \\
(0.006)\end{array}$ & $\begin{array}{c}-0.873^{* * *} \\
(0.007)\end{array}$ & $\begin{array}{c}-0.841^{* * *} \\
(0.007)\end{array}$ & $\begin{array}{c}-0.864^{* * *} \\
(0.007)\end{array}$ & $\begin{array}{c}-0.908^{* * *} \\
(0.007)\end{array}$ \\
\hline Post $*$ Ingresos altos & $\begin{array}{c}-0.729^{* * *} \\
(0.009)\end{array}$ & $\begin{array}{c}-0.768^{* * *} \\
(0.009)\end{array}$ & $\begin{array}{c}-0.793^{* * *} \\
(0.01)\end{array}$ & $\begin{array}{c}-0.759^{* * *} \\
(0.01)\end{array}$ & $\begin{array}{c}-0.777^{* * *} \\
(0.011)\end{array}$ & $\begin{array}{c}-0.828^{* * *} \\
(0.011)\end{array}$ \\
\hline Post * Ingresos muy altos & $\begin{array}{c}-0.351^{* * *} \\
(0.01)\end{array}$ & $\begin{array}{c}-0.386^{* * *} \\
(0.011)\end{array}$ & $\begin{array}{c}-0.419^{* * *} \\
(0.011)\end{array}$ & $\begin{array}{c}-0.381^{* * *} \\
(0.011)\end{array}$ & $\begin{array}{c}-0.413^{* * *} \\
(0.012)\end{array}$ & $\begin{array}{c}-0.463^{* * *} \\
(0.012)\end{array}$ \\
\hline Observaciones & 293,680 & 293,680 & 293,680 & 293,680 & 293,680 & 293,680 \\
\hline R-cuadrado & 0.183 & 0.184 & 0.174 & 0.171 & 0.145 & 0.160 \\
\hline Variedades & 70,294 & 70,294 & 70,294 & 70,294 & 70,294 & 70,294 \\
\hline
\end{tabular}

Los coeficientes estimados arrojan un resultado interesante: la reducción en la calidad parece ser algo mayor para las variedades que provienen de países con menores ingresos per cápita, y menor para las variedades que provienen de países con mayores ingresos. 
Este resultado también es robusto respecto a las distintas medidas de calidad utilizadas.

\subsection{Cambio en el set de importados}

Por último, se intenta corroborar la hipótesis final de sustitución de variedades. La tabla 5 presenta las estimaciones del coeficiente de interés $(\beta)$ que acompaña a la interacción de la variable Post con la calidad estimada ${ }^{11}$ (Panel A) y la interacción de variable Post con los precios (Panel B). En las primeras tres columnas, la variable dependiente es la participación de cada variedad en la categoría de producto a la que pertenece (sustitución a nivel producto); y en las columnas 4 a 6 es la participación de cada producto en la industria (sustitución a nivel industria). El panel C incluye ambas interacciones a la vez.

Los coeficientes negativos y significativos estimados en el panel A siguen la línea de las hipótesis planteadas: en promedio, las variedades de mayor (menor) calidad pierden (ganan) participación en la canasta de consumo ${ }^{12}$. La sustitución comienza ya en el año 2000, pero su magnitud aumenta considerablemente y se concentra en el año de la crisis cambiaria. Los resultados a nivel producto refuerzan este argumento. En el caso de los precios (panel B), un resultado que incomoda es el signo positivo y significativo para el año 2002. Sin embargo, el resultado esperado (sustitución hacia variedades de menor precio) ocurre en los años posteriores (2003 y 2004). Al comparar los 3 años previos a la crisis con los 3 años posteriores (columna 1 del panel B), prevalece el resultado de sustitución esperado. A nivel producto, ninguno de los coeficientes estimados es estadísticamente significativo.

\footnotetext{
${ }^{11}$ Se utiliza la definición más completa de calidad (6) estimada por VI. Los resultados son robustos a las demás medidas de calidad.

${ }^{12}$ En el caso de la calidad, los resultados no varían al dejar fija la calidad en un año base determinado (1999, 2000 o 2001).
} 
Tabla 5. Sustitución hacia variedades y productos de menor calidad y menor precio

\begin{tabular}{|c|c|c|c|c|c|c|}
\hline & \multicolumn{3}{|c|}{ Share de la Variedad } & \multicolumn{3}{|c|}{ Share del Producto } \\
\hline & (1) & $(2)$ & (3) & (4) & (5) & $(6)$ \\
\hline \multicolumn{7}{|l|}{ Panel A: Calidad } \\
\hline Post $*$ Calidad & $\begin{array}{c}-0.140^{* * *} \\
(0.025)\end{array}$ & $\begin{array}{c}-0.146^{* * *} \\
(0.029)\end{array}$ & & $\begin{array}{c}-0.078 \\
(0.05)\end{array}$ & $\begin{array}{c}-0.202^{* * *} \\
(0.067)\end{array}$ & \\
\hline Año $2000 *$ Calidad & & & $\begin{array}{c}-0.062^{* *} \\
(0.025)\end{array}$ & & & $\begin{array}{c}0.003 \\
(0.057)\end{array}$ \\
\hline Año $2001 *$ Calidad & & & $\begin{array}{c}-0.049^{*} \\
(0.028)\end{array}$ & & & $\begin{array}{c}-0.090^{*} \\
(0.054)\end{array}$ \\
\hline Año $2002 *$ Calidad & & & $\begin{array}{c}-0.320^{* * *} \\
(0.038)\end{array}$ & & & $\begin{array}{c}-0.254^{* * *} \\
(0.076)\end{array}$ \\
\hline Año $2003 *$ Calidad & & & $\begin{array}{l}-0.009 \\
(0.036)\end{array}$ & & & $\begin{array}{l}-0.117 \\
(0.073)\end{array}$ \\
\hline Año $2004 *$ Calidad & & & $\begin{array}{c}0.137^{* * *} \\
(0.036)\end{array}$ & & & $\begin{array}{l}0.061 \\
(0.07)\end{array}$ \\
\hline Observaciones & 293,680 & 180,808 & 293,680 & 38,097 & 24,763 & 38,097 \\
\hline R-cuadrado & 0.055 & 0.056 & 0.075 & 0.009 & 0.010 & 0.011 \\
\hline Variedades & 70,294 & 57,668 & 70,294 & & & \\
\hline Productos & & & & 7,400 & 6,967 & 7,400 \\
\hline \multicolumn{7}{|l|}{ Panel B: Precio } \\
\hline Post $*$ Precio & $\begin{array}{c}-0.101^{* * *} \\
(0.027)\end{array}$ & $\begin{array}{c}-0.012 \\
(0.03)\end{array}$ & & $\begin{array}{l}-0.040 \\
(0.042)\end{array}$ & $\begin{array}{l}-0.001 \\
(0.048)\end{array}$ & \\
\hline Año $2000 *$ Precio & & & $\begin{array}{c}0.023 \\
(0.027)\end{array}$ & & & $\begin{array}{l}0.003 \\
(0.04)\end{array}$ \\
\hline Año $2001 *$ Precio & & & $\begin{array}{c}-0.033 \\
(0.03)\end{array}$ & & & $\begin{array}{c}0.003 \\
(0.046)\end{array}$ \\
\hline Año $2002 *$ Precio & & & $\begin{array}{c}0.091^{* *} \\
(0.039)\end{array}$ & & & $\begin{array}{c}0.002 \\
(0.061)\end{array}$ \\
\hline Año $2003 *$ Precio & & & $\begin{array}{c}-0.131^{* * *} \\
(0.038)\end{array}$ & & & $\begin{array}{l}-0.017 \\
(0.056)\end{array}$ \\
\hline Año $2004 *$ Precio & & & $\begin{array}{c}-0.228^{* * *} \\
(0.037)\end{array}$ & & & $\begin{array}{l}-0.086 \\
(0.055)\end{array}$ \\
\hline Observaciones & 297,244 & 182,799 & 297,244 & 38,220 & 24,807 & 38,220 \\
\hline R-cuadrado & 0.034 & 0.036 & 0.035 & 0.034 & 0.037 & 0.034 \\
\hline Variedades & 71,061 & 58,291 & 71,061 & & & \\
\hline Productos & & & & 7,425 & 6,988 & 7,425 \\
\hline EF variedad & $\mathrm{Si}$ & $\mathrm{Si}$ & $\mathrm{Si}$ & & & \\
\hline EF Producto & & & & $\mathrm{Si}$ & $\mathrm{Si}$ & $\mathrm{Si}$ \\
\hline
\end{tabular}

Columnas (1), (3), (4), (6): período 1999 a 2004.

Columnas (2), (5): período 2000 a 2003.

Finalmente, en el panel $\mathrm{C}$ se tiene en cuenta sustitución por calidad y por precio de manera conjunta. En este caso, también prevalece la hipótesis planteada: los coeficientes estimados indican que en promedio, las variedades de mayor (menor) precio pierden (ganan) participación en la canasta de consumo en los años posteriores a la crisis cambiaria. Del análisis 
de los resultados, surge que la sustitución por calidad es un resultado robusto a todas las especificaciones estimadas, mientras que la sustitución por precio es un resultado que debe ser tomado con mayor precaución.

Tabla 5. Continuación.

\begin{tabular}{|c|c|c|c|c|c|c|}
\hline & \multicolumn{3}{|c|}{ Share de la Variedad } & \multicolumn{3}{|c|}{ Share del Producto } \\
\hline & (1) & $(2)$ & (3) & (4) & (5) & (6) \\
\hline \multicolumn{7}{|c|}{ Panel C: Calidad y Precio } \\
\hline Post $*$ Precio & $\begin{array}{c}-0.388^{* * *} \\
(0.035)\end{array}$ & $\begin{array}{c}-0.171^{* * *} \\
(0.04)\end{array}$ & & $\begin{array}{l}-0.043 \\
(0.045)\end{array}$ & $\begin{array}{l}-0.019 \\
(0.052)\end{array}$ & \\
\hline Post $*$ Calidad & $\begin{array}{c}-0.371^{* * *} \\
(0.033)\end{array}$ & $\begin{array}{c}-0.262^{* * *} \\
(0.038)\end{array}$ & & $\begin{array}{c}-0.089^{*} \\
(0.054)\end{array}$ & $\begin{array}{c}-0.189 * * \\
(0.073)\end{array}$ & \\
\hline Observations & 293,680 & 180,808 & & 38,097 & 24,763 & \\
\hline R-squared & 0.072 & 0.073 & & 0.038 & 0.041 & \\
\hline Varieties & 70,294 & 57,668 & & & & \\
\hline Products & & & & 7,400 & 6,967 & \\
\hline EF variedad & $\mathrm{Si}$ & $\mathrm{Si}$ & $\mathrm{Si}$ & & & \\
\hline EF Producto & & & & $\mathrm{Si}$ & $\mathrm{Si}$ & $\mathrm{Si}$ \\
\hline
\end{tabular}

Columnas (1), (3), (4), (6): período 1999 a 2004.

Columnas (2), (5): período 2000 a 2003.

\section{Comentarios finales}

Existe una amplia literatura dedicada a estudiar el traspaso a precios de las variaciones en el tipo de cambio (pass-through). Existe consenso en que el traslado a precios es rezagado e incompleto, y varía considerablemente entre las diferentes industrias, países y a lo largo del tiempo. La literatura ha presentado diversas explicaciones, tanto teóricas como empíricas, para comprender las causas del pass-through incompleto: presencia de bienes no transables en la canasta de consumo, existencia de costos de distribución en moneda local, poder de mercado de las firmas domésticas e internacionales que conlleva a la determinación estratégica de los márgenes de ganancia, rigideces nominales de precios y costos de ajuste.

En este trabajo se estudia la racionalidad de un canal (complementario a los anteriores) que no ha sido explorado en la literatura: el fenómeno de sustitución de variedades. Es bien sabido que la devaluación genera una caída en el ingreso real de los consumidores. La evidencia aquí presentada muestra que como consecuencia de la disminución del ingreso real, se reduce la disposición a pagar de parte de los consumidores. Las estimaciones de calidad realizadas revelan que la calidad de las variedades y productos importados disminuye en los años que siguen a la crisis cambiaria argentina. La caída en la calidad resulta ser decreciente en el nivel de ingresos del país de origen. Las últimas estimaciones siguen la línea de nuestro argumento, evidenciando un cambio composicional en la canasta de importaciones, donde las variedades de mayor (menor) calidad y mayor (menor) precio 
pierden (ganan) participación en la canasta de consumo. Como consecuencia, esta sustitución produce una caída en los precios promedio de las variedades y productos importados, que actúa como un atenuante adicional de la suba de precios de estos mismos productos expresados en moneda local. 


\section{Referencias}

Amiti, M., Itskhoki, O. y Konings, J. (2014). "Importers, Exporters, and Exchange Rate Disconnect," American Economic Review, American Economic Association, 104(7): 1942-78.

Atkeson, A. y Burstein, A. (2008). "Pricing-to-Market, Trade Costs, and International Relative Prices," American Economic Review, 98(5): 1998-2031.

Bacchetta, P. Y VAn Wincoop, E. (2003. "Why Do Consumer Prices React Less Than Import Prices to Exchange Rates?," Journal of the European Economic Association, MIT Press, 1(2-3): 662-670.

Bergin, P. y Feenstra, R. (2001). "Pricing-to-market, Staggered Contracts, and Real Exchange Rate Persistence," Journal of International Economics, Elsevier, 54(2): 333-359.

Burstein, A. y Gopinath, R. (2013). "International Prices and Exchange Rates," Handbook of International Economics, 4.

Burstein, A., Eichenbaum, M. y Rebelo, S. (2005). "Large Devaluations and the Real Exchange Rate," Journal of Political Economy, University of Chicago Press, 113(4): 742-784.

Burstein, A., Neves, J. y Rebelo, S. (2003). "Distribution Costs and Real Exchange Rate Dynamics During Exchange-rate-based Stabilizations," Journal of Monetary Economics, Elsevier, 50(6): 1189-1214.

Campa, J.M., Y Goldberg, L. (2005). "Exchange Rate Pass-Through into Import Prices," The Review of Economics and Statistics, MIT Press, 87(4): 679-690.

Campa, J.M., Y GoldberG, L. (2006). "Distribution Margins, Imported Inputs, and the Sensitivity of the CPI to Exchange Rates," NBER Working Papers 12121.

Campa, J.M., y Goldberg, L. (2010). "The Sensitivity of the CPI to Exchange Rates: Distribution Margins, Imported Inputs, and Trade Exposure," The Review of Economics and Statistics, 92(2): 392-407.

Campos, C. (2010). "Incomplete Exchange Rate Pass-Through and Extensive Margin of Adjustment," Department of Economics, Yale University.

Corsetti, G., y Dedola, L. (2004). "Macroeconomics of International Price Discrimination," Journal of International Economics, 67(1): 129-155.

Devereux, M. B., y Engel . C. (2002). "Exchange Rate Pass-through, Exchange Rate Volatility, and Exchange Rate Disconnect," Journal of Monetary Economics, 49: 913-940.

Dornbusch, R. (1987). "Exchange Rates and Prices," American Economic Review, 77: 93-106.

Engel, C. (1993). "Real Exchange Rates and Relative Prices," Journal of Monetary Economics, 32(1): 35-50.

Gopinath, G. y O. Itskhoki (2010). "Frequency of Price Adjustment and Pass-Through," The Quarterly Journal of Economics, 125(2): 675-727.

Gopinath, G. y Neiman, B. (2014). "Trade Adjustment and Productivity in Large Crises," American Economic Review, 104(3): 793-831.

Hellerstein, R. (2004). "Who Bears the Cost of a Change in the Exchange Rate? The Case of Imported Beer," Staff Reports 179, Federal Reserve Bank of New York.

Hooper, P. y Mann, C. (1989). "Exchange Rate Pass-Through in the 1980s: The Case of U.S. Imports of Manufactures," Brookings Papers on Economic Activity, Economic Studies Program, The Brookings Institution, 20(1): 297-337. 
Marston, R. (1990). "Pricing to market in Japanese manufacturing," Journal of International Economics, 29(3-4): 217-236.

McKenzie, D. y Schargrodsky, E. (2005). "Buying Less, But Shopping More: Changes In Consumption Patterns During A Crisis," Escuela de Negocios, Universidad Torcuato Di Tella, Documento de trabajo 01/2005.

Nakamura, E. y Zerom, D. (2010). "Accounting for Incomplete Pass-Through," Review of Economic Studies, Wiley Blackwell, 77(3): 1192-1230.

Nakamura, E. (2008). "Pass-Through in Retail and Wholesale," American Economic Review, American Economic Association, 98(2): 430-37.

Ohno, K. (1989). "Export Pricing Behavior of Manufacturing: A U.S.-Japan Comparison," IMF Staff Papers, 36(3): 550-79.

PARsley, D. C., Y Wei, S-J. (2001). "Explaining the Border Effect: The Role of Exchange Rate Variability, Shipping Costs and Geography," Journal of International Economics, 55: $87-105$.

Khandelwal, A. (2010). "The Long and Short (of) Quality Ladders," Review of Economic Studies, 77(4): 1450-1476.

Knetter, M. (1989). "Price Discrimination by U.S. and German Exporters," American Economic Review, American Economic Association, 79(1): 198-210.

Kravis, I. y Lipsey, R. (1978). "Price Behavior in the Light of Balance of PAyments Theories," Journal of International Economics, Elsevier, 8(2): 193-246.

Kreinin, M. E. (1977). "The Effect of Exchange Rate Changes on the Prices and Volume of Foreign Trade," International Monetary Fund Staff Papers, 24(2): 297-329.

Woo, W. T. (1984). "Exchange Rates and the Prices of Nonfood, Nonfuel Products," Brookings Papers on Economic Activivt, 2: 511-530. 


\section{Anexo}

Figura A.1:

Cambio en el logaritmo del precio de variedades importadas. 2002-2000 Sin ponderadores.

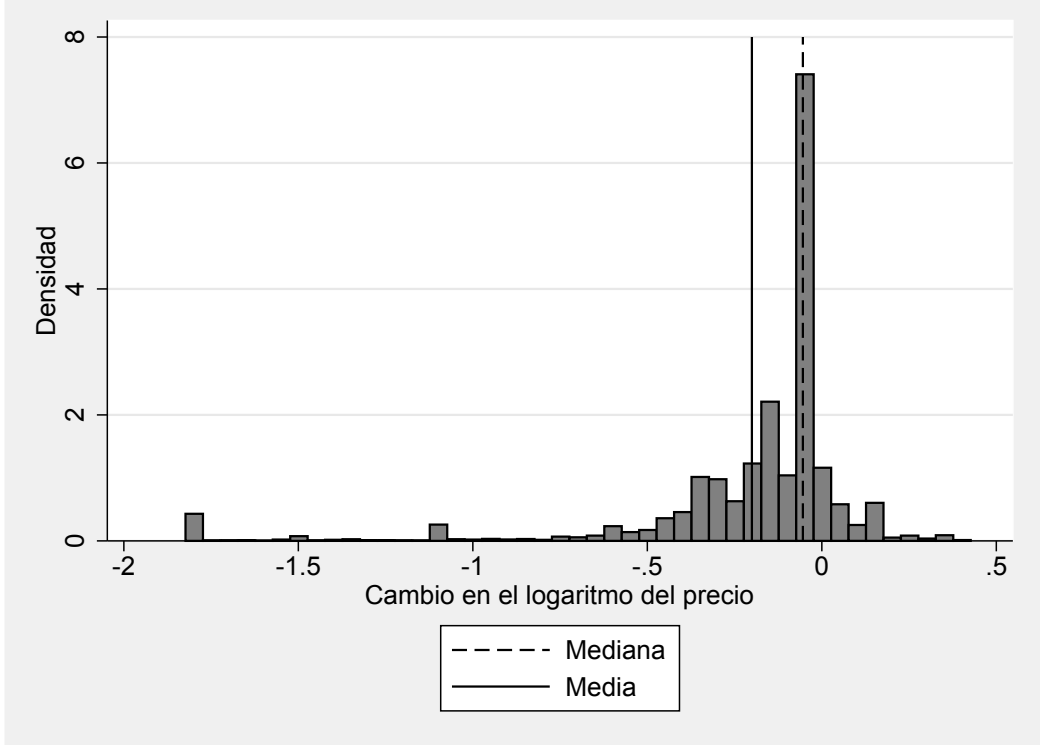

Por cuestiones de exposición se excluyen el percentil 1 y el percentil 99.

Figura A.2:

Cambio en el logaritmo del precio de variedades importadas. 2002-2000 Ponderando por cantidades importadas.

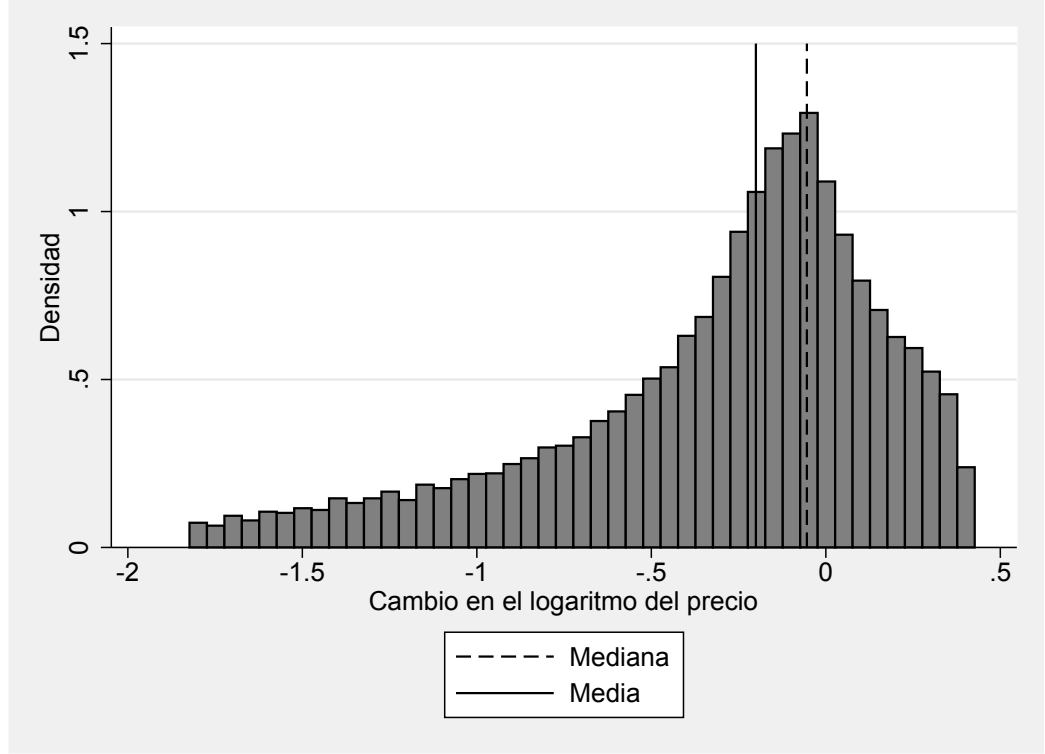

Por cuestiones de exposición se excluyen el percentil 1 y el percentil 99. 
Figura A.3:

Cambio en el logaritmo del precio de productos importados. 2002-2000 Sin ponderadores.

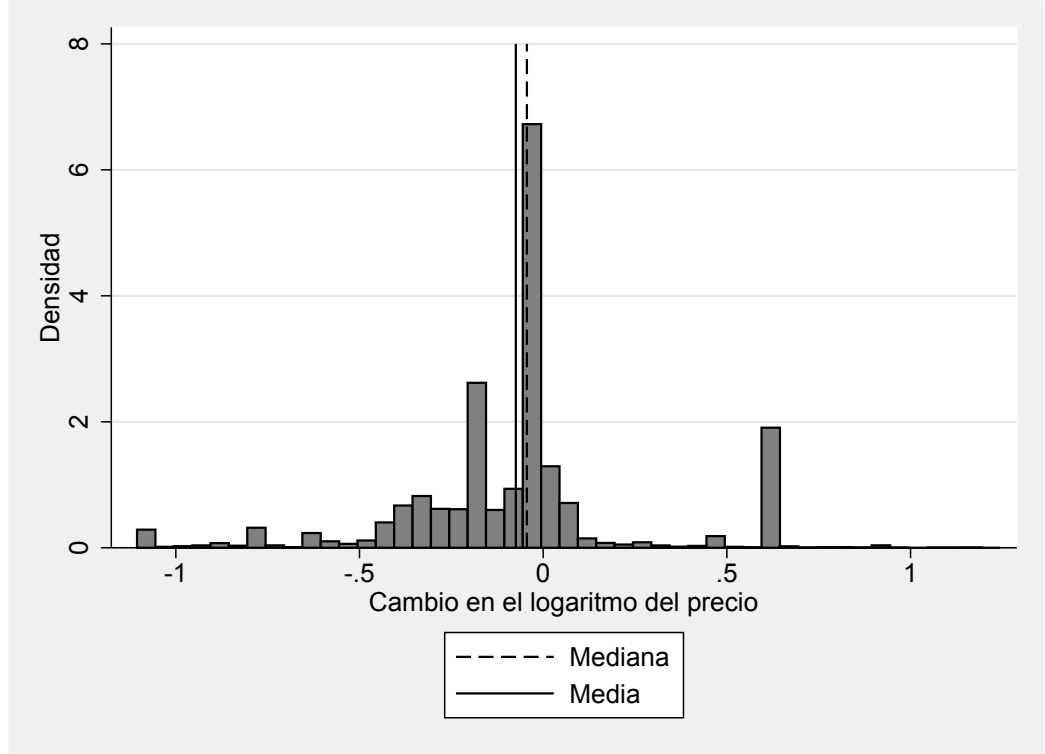

Por cuestiones de exposición se excluyen el percentil 1 y el percentil 99.

Figura A.4:

Cambio en el logaritmo del precio de productos importados. 2002-2000 Ponderando por cantidades importadas.

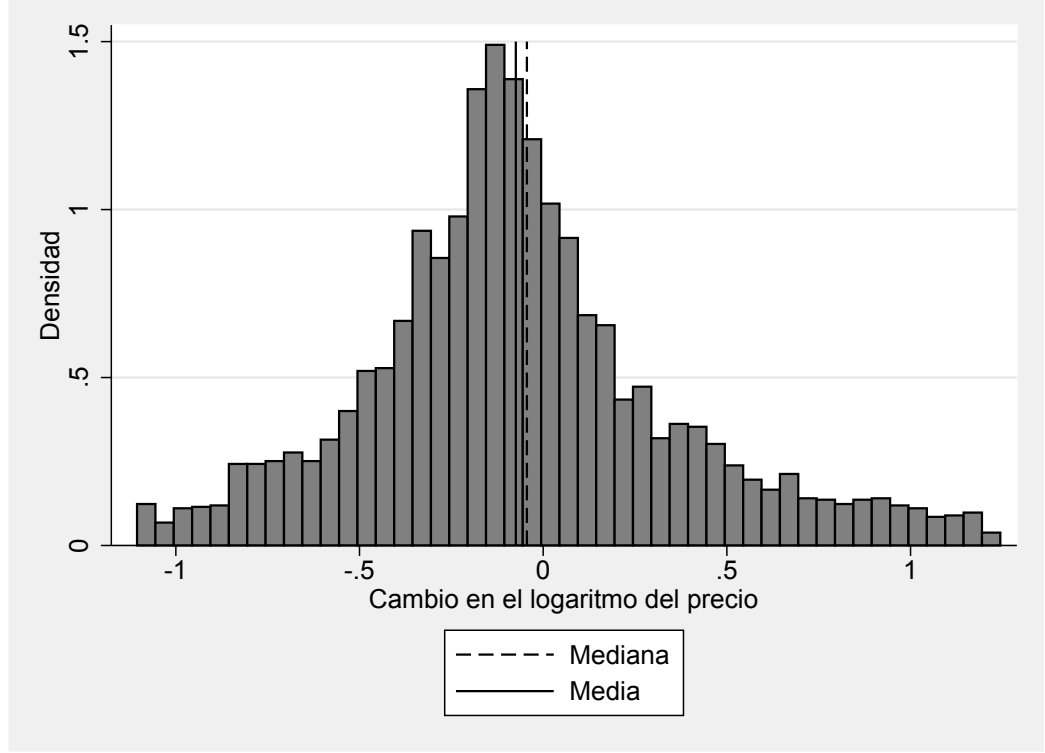

Por cuestiones de exposición se excluyen el percentil 1 y el percentil 99. 
Tabla A1. Clasificación de industrias

\begin{tabular}{llc}
\hline \hline & Nombre & Categorías a 2 dígitos \\
\hline 1 & Alimentos manufacturados & $(16-24)$ \\
2 & Productos minerales & $(25-27)$ \\
3 & Químicos & $(28-38)$ \\
4 & Plásticos y cauchos & $(39-40)$ \\
5 & Productos de cuero y pieles & $(41-43)$ \\
6 & Productos de madera & $(44-49)$ \\
7 & Textiles & $(50-63)$ \\
8 & Calzado y sombreros & $(64-67)$ \\
9 & Productos de piedra y vidrio & $(68-71)$ \\
10 & Metales & $(72-83)$ \\
11 & Maquinaria y maquinaria eléctrica & $(84-85)$ \\
12 & Equipos de transporte & $(86-89)$ \\
13 & Varios & $(90-97)$ \\
\hline
\end{tabular}


Tabla A2. Parámetros de la Demanda. MCO

\begin{tabular}{|c|c|c|c|c|c|c|}
\hline & \multicolumn{6}{|c|}{ Log Share de la Variedad Normalizado } \\
\hline & (1) & (2) & $(3)$ & (4) & (5) & (6) \\
\hline Precio & $\begin{array}{c}-0.121^{* * *} \\
(0.003)\end{array}$ & & & & & \\
\hline Precio 1 & & $\begin{array}{c}-0.119^{* * *} \\
(0.028)\end{array}$ & $\begin{array}{c}-0.100^{* * *} \\
(0.023)\end{array}$ & $\begin{array}{c}-0.121^{* * *} \\
(0.028)\end{array}$ & $\begin{array}{c}-0.368^{* * *} \\
(0.017)\end{array}$ & $\begin{array}{c}-0.100^{* * *} \\
(0.023)\end{array}$ \\
\hline Precio 2 & & $\begin{array}{c}-0.264^{* * *} \\
(0.043)\end{array}$ & $\begin{array}{c}-0.255^{* * *} \\
(0.042)\end{array}$ & $\begin{array}{c}-0.267^{* * *} \\
(0.043)\end{array}$ & $\begin{array}{c}-0.760^{* * *} \\
(0.05)\end{array}$ & $\begin{array}{c}-0.258^{* * *} \\
(0.042)\end{array}$ \\
\hline Precio 3 & & $\begin{array}{c}-0.171^{* * *} \\
(0.007)\end{array}$ & $\begin{array}{c}-0.153^{* * *} \\
(0.006)\end{array}$ & $\begin{array}{c}-0.171^{* * *} \\
(0.007)\end{array}$ & $\begin{array}{c}-0.648^{* * *} \\
(0.011)\end{array}$ & $\begin{array}{c}-0.154^{* * *} \\
(0.006)\end{array}$ \\
\hline Precio 4 & & $\begin{array}{c}-0.152^{* * *} \\
(0.009)\end{array}$ & $\begin{array}{c}-0.146^{* * *} \\
(0.009)\end{array}$ & $\begin{array}{c}-0.153^{* * *} \\
(0.009)\end{array}$ & $\begin{array}{c}-0.858^{* * *} \\
(0.019)\end{array}$ & $\begin{array}{c}-0.147^{* * *} \\
(0.009)\end{array}$ \\
\hline Precio 5 & & $\begin{array}{c}-0.113^{* * *} \\
(0.018)\end{array}$ & $\begin{array}{c}-0.089^{* * *} \\
(0.013)\end{array}$ & $\begin{array}{c}-0.111^{* * *} \\
(0.018)\end{array}$ & $\begin{array}{c}-0.756^{* * *} \\
(0.026)\end{array}$ & $\begin{array}{c}-0.089^{* * *} \\
(0.013)\end{array}$ \\
\hline Precio 6 & & $\begin{array}{c}-0.106^{* * *} \\
(0.015)\end{array}$ & $\begin{array}{c}-0.116^{* * *} \\
(0.012)\end{array}$ & $\begin{array}{c}-0.106^{* * *} \\
(0.015)\end{array}$ & $\begin{array}{c}-0.951^{* * *} \\
(0.019)\end{array}$ & $\begin{array}{c}-0.116^{* * *} \\
(0.012)\end{array}$ \\
\hline Precio 7 & & $\begin{array}{c}-0.137^{* * *} \\
(0.012)\end{array}$ & $\begin{array}{c}-0.146^{* * *} \\
(0.012)\end{array}$ & $\begin{array}{c}-0.143^{* * *} \\
(0.012)\end{array}$ & $\begin{array}{c}-0.960^{* * *} \\
(0.024)\end{array}$ & $\begin{array}{c}-0.147^{* * *} \\
(0.012)\end{array}$ \\
\hline Precio 8 & & $\begin{array}{c}0.012 \\
(0.025)\end{array}$ & $\begin{array}{c}-0.044^{* *} \\
(0.02)\end{array}$ & $\begin{array}{c}0.014 \\
(0.024)\end{array}$ & $\begin{array}{c}-0.628^{* * *} \\
(0.04)\end{array}$ & $\begin{array}{c}-0.043^{* *} \\
(0.02)\end{array}$ \\
\hline Precio 9 & & $\begin{array}{c}-0.158^{* * *} \\
(0.013)\end{array}$ & $\begin{array}{c}-0.144^{* * *} \\
(0.012)\end{array}$ & $\begin{array}{c}-0.156^{* * *} \\
(0.012)\end{array}$ & $\begin{array}{c}-0.830^{* * *} \\
(0.019)\end{array}$ & $\begin{array}{c}-0.144^{* * * *} \\
(0.012)\end{array}$ \\
\hline Precio 10 & & $\begin{array}{c}-0.161^{* * *} \\
(0.007)\end{array}$ & $\begin{array}{c}-0.147^{* * *} \\
(0.007)\end{array}$ & $\begin{array}{c}-0.160^{* * *} \\
(0.007)\end{array}$ & $\begin{array}{c}-0.928^{* * *} \\
(0.016)\end{array}$ & $\begin{array}{c}-0.148^{* * *} \\
(0.007)\end{array}$ \\
\hline Precio 11 & & $\begin{array}{c}-0.098^{* * *} \\
(0.003)\end{array}$ & $\begin{array}{c}-0.090^{* * *} \\
(0.003)\end{array}$ & $\begin{array}{c}-0.095^{* * *} \\
(0.003)\end{array}$ & $\begin{array}{c}-0.727^{* * *} \\
(0.003)\end{array}$ & $\begin{array}{c}-0.090^{* * *} \\
(0.003)\end{array}$ \\
\hline Precio 12 & & $\begin{array}{c}-0.126^{* * *} \\
(0.019)\end{array}$ & $\begin{array}{c}-0.130^{* * *} \\
(0.017)\end{array}$ & $\begin{array}{c}-0.125^{* * *} \\
(0.019)\end{array}$ & $\begin{array}{c}-0.905^{* * *} \\
(0.009)\end{array}$ & $\begin{array}{c}-0.129^{* * *} \\
(0.017)\end{array}$ \\
\hline Precio 13 & & $\begin{array}{c}-0.109^{* * *} \\
(0.005)\end{array}$ & $\begin{array}{c}-0.098^{* * *} \\
(0.005)\end{array}$ & $\begin{array}{c}-0.108^{* * *} \\
(0.005)\end{array}$ & $\begin{array}{c}-0.733^{* * *} \\
(0.006)\end{array}$ & $\begin{array}{c}-0.099^{* * *} \\
(0.005)\end{array}$ \\
\hline Precio * Post & $\begin{array}{c}-0.012^{* * *} \\
(0.002)\end{array}$ & $\begin{array}{c}-0.012^{* * *} \\
(0.002)\end{array}$ & $\begin{array}{c}-0.023^{* * *} \\
(0.002)\end{array}$ & $\begin{array}{c}-0.019^{* * *} \\
(0.002)\end{array}$ & $\begin{array}{c}-0.028^{* * *} \\
(0.005)\end{array}$ & $\begin{array}{c}-0.025^{* * *} \\
(0.002)\end{array}$ \\
\hline Sigma & $\begin{array}{c}0.881^{* * *} \\
(0.002)\end{array}$ & $\begin{array}{c}0.879^{* * *} \\
(0.002)\end{array}$ & $\begin{array}{c}0.882^{* * *} \\
(0.002)\end{array}$ & $\begin{array}{c}0.879^{* * *} \\
(0.002)\end{array}$ & $\begin{array}{c}0.595^{* * *} \\
(0.016)\end{array}$ & $\begin{array}{c}0.881^{* * *} \\
(0.002)\end{array}$ \\
\hline Observaciones & 297,244 & 297,244 & 297,244 & 297,244 & 325,449 & 297,244 \\
\hline R-cuadrado & 0.852 & 0.852 & 0.877 & 0.854 & 0.698 & 0.878 \\
\hline Variedades & 71,061 & 71,061 & 71,061 & 71,061 & & 71,061 \\
\hline EF año & $\mathrm{Si}$ & $\mathrm{Si}$ & No & No & No & No \\
\hline EF año-industria & No & No & $\mathrm{Si}$ & No & $\mathrm{Si}$ & $\mathrm{Si}$ \\
\hline EF año-país & No & No & No & $\mathrm{Si}$ & $\mathrm{Si}$ & $\mathrm{Si}$ \\
\hline EF producto-país & $\mathrm{Si}$ & $\mathrm{Si}$ & $\mathrm{Si}$ & $\mathrm{Si}$ & No & $\mathrm{Si}$ \\
\hline
\end{tabular}


Tabla A3. Parámetros de la Demanda. VI

\begin{tabular}{|c|c|c|c|c|c|c|}
\hline & \multicolumn{6}{|c|}{ Log Share de la Variedad Normalizado } \\
\hline & (1) & $(2)$ & $(3)$ & (4) & $(5)$ & (6) \\
\hline Precio & $\begin{array}{c}-0.370^{* * *} \\
(0.041)\end{array}$ & & & & & \\
\hline Precio 1 & & $\begin{array}{c}-2.566^{* * *} \\
(0.337)\end{array}$ & $\begin{array}{c}-3.014^{* * *} \\
(0.371)\end{array}$ & $\begin{array}{c}-2.937^{* * *} \\
(0.395)\end{array}$ & $\begin{array}{c}-1.285^{* * *} \\
(0.109)\end{array}$ & $\begin{array}{c}-3.679^{* * *} \\
(0.513)\end{array}$ \\
\hline Precio 2 & & $\begin{array}{c}-0.782^{* * *} \\
(0.117)\end{array}$ & $\begin{array}{c}-0.977^{* * *} \\
(0.124)\end{array}$ & $\begin{array}{c}-0.921^{* * *} \\
(0.135)\end{array}$ & $\begin{array}{c}-1.205^{* * *} \\
(0.14)\end{array}$ & $\begin{array}{c}-1.213^{* * *} \\
(0.17)\end{array}$ \\
\hline Precio 3 & & $\begin{array}{c}-0.618^{* * *} \\
(0.071)\end{array}$ & $\begin{array}{c}-0.781 * * * \\
(0.077)\end{array}$ & $\begin{array}{c}-0.738^{* * *} \\
(0.089)\end{array}$ & $\begin{array}{c}-0.836^{* * *} \\
(0.034)\end{array}$ & $\begin{array}{c}-0.983^{* * *} \\
(0.121)\end{array}$ \\
\hline Precio 4 & & $\begin{array}{c}-0.623^{* * *} \\
(0.077)\end{array}$ & $\begin{array}{c}-0.806^{* * *} \\
(0.084)\end{array}$ & $\begin{array}{c}-0.740^{* * *} \\
(0.096)\end{array}$ & $\begin{array}{c}-1.177^{* * *} \\
(0.055)\end{array}$ & $\begin{array}{c}-1.015^{* * *} \\
(0.132)\end{array}$ \\
\hline Precio 5 & & $\begin{array}{c}-0.366^{* * *} \\
(0.052)\end{array}$ & $\begin{array}{c}-0.426^{* * *} \\
(0.054)\end{array}$ & $\begin{array}{c}-0.427^{* * *} \\
(0.06)\end{array}$ & $\begin{array}{c}-1.151^{* * *} \\
(0.057)\end{array}$ & $\begin{array}{c}-0.530^{* * *} \\
(0.076)\end{array}$ \\
\hline Precio 6 & & $\begin{array}{c}-0.486^{* * *} \\
(0.066)\end{array}$ & $\begin{array}{c}-0.687^{* * *} \\
(0.07)\end{array}$ & $\begin{array}{c}-0.585^{* * *} \\
(0.08)\end{array}$ & $\begin{array}{c}-1.400^{* * *} \\
(0.061)\end{array}$ & $\begin{array}{c}-0.851^{* * *} \\
(0.106)\end{array}$ \\
\hline Precio 7 & & $\begin{array}{c}-0.697^{* * *} \\
(0.062)\end{array}$ & $\begin{array}{c}-0.855^{* * *} \\
(0.068)\end{array}$ & $\begin{array}{c}-0.803^{* * *} \\
(0.076)\end{array}$ & $\begin{array}{c}-1.418^{* * *} \\
(0.049)\end{array}$ & $\begin{array}{c}-1.019^{* * *} * \\
(0.104)\end{array}$ \\
\hline Precio 8 & & $\begin{array}{c}-0.367^{* * *} \\
(0.087)\end{array}$ & $\begin{array}{c}-0.634^{* * *} \\
(0.096)\end{array}$ & $\begin{array}{c}-0.460^{* * *} \\
(0.102)\end{array}$ & $\begin{array}{c}-1.345^{* * *} \\
(0.067)\end{array}$ & $\begin{array}{c}-0.799^{* * *} \\
(0.132)\end{array}$ \\
\hline Precio 9 & & $\begin{array}{c}-0.441^{* * *} \\
(0.05)\end{array}$ & $\begin{array}{c}-0.557^{* * *} \\
(0.054)\end{array}$ & $\begin{array}{c}-0.515^{* * *} \\
(0.061)\end{array}$ & $\begin{array}{c}-1.022^{* * *} \\
(0.036)\end{array}$ & $\begin{array}{c}-0.688^{* * *} * \\
(0.083)\end{array}$ \\
\hline Precio 10 & & $\begin{array}{c}-0.461^{* * *} \\
(0.052)\end{array}$ & $\begin{array}{c}-0.598^{* * *} \\
(0.057)\end{array}$ & $\begin{array}{c}-0.543^{* * *} \\
(0.065)\end{array}$ & $\begin{array}{c}-1.163^{* * *} \\
(0.049)\end{array}$ & $\begin{array}{c}-0.743^{* * *} \\
(0.09)\end{array}$ \\
\hline Precio 11 & & $\begin{array}{c}-0.257^{* * *} \\
(0.029)\end{array}$ & $\begin{array}{c}-0.326^{* * *} \\
(0.031)\end{array}$ & $\begin{array}{c}-0.302^{* * *} \\
(0.036)\end{array}$ & $\begin{array}{c}-0.798^{* * *} \\
(0.007)\end{array}$ & $\begin{array}{c}-0.407^{* * *} \\
(0.05)\end{array}$ \\
\hline Precio 12 & & $\begin{array}{c}-0.365^{* * *} \\
(0.056)\end{array}$ & $\begin{array}{c}-0.522^{* * *} \\
(0.062)\end{array}$ & $\begin{array}{c}-0.452^{* * *} \\
(0.068)\end{array}$ & $\begin{array}{c}-0.958^{* * *} \\
(0.016)\end{array}$ & $\begin{array}{c}-0.666^{* * *} \\
(0.092)\end{array}$ \\
\hline Precio 13 & & $\begin{array}{c}-0.278^{* * *} \\
(0.031)\end{array}$ & $\begin{array}{c}-0.347^{* * *} \\
(0.033)\end{array}$ & $\begin{array}{c}-0.328^{* * *} \\
(0.039)\end{array}$ & $\begin{array}{c}-0.824^{* * *} \\
(0.013)\end{array}$ & $\begin{array}{c}-0.434^{* * *} \\
(0.053)\end{array}$ \\
\hline Precio * Post & $\begin{array}{c}-0.022^{* * *} \\
(0.002)\end{array}$ & $\begin{array}{c}-0.019^{* * *} \\
(0.002)\end{array}$ & $\begin{array}{c}-0.019^{* * *} \\
(0.002)\end{array}$ & $\begin{array}{c}-0.026^{* * *} \\
(0.002)\end{array}$ & $\begin{array}{c}-0.030^{* * *} \\
(0.008)\end{array}$ & $\begin{array}{c}-0.017^{* * *} * \\
(0.004)\end{array}$ \\
\hline Sigma & $\begin{array}{c}0.755^{* * *} \\
(0.032)\end{array}$ & $\begin{array}{c}0.740^{* * *} \\
(0.034)\end{array}$ & $\begin{array}{c}0.653^{* * *} \\
(0.036)\end{array}$ & $\begin{array}{c}0.682^{* * *} \\
(0.042)\end{array}$ & $\begin{array}{c}0.561^{* * *} \\
(0.051)\end{array}$ & $\begin{array}{c}0.556^{* * *} \\
(0.058)\end{array}$ \\
\hline Observaciones & 293,680 & 293,680 & 293,680 & 293,680 & 321,645 & 293,680 \\
\hline R-cuadrado & 0.832 & 0.810 & 0.800 & 0.789 & 0.689 & 0.745 \\
\hline Variedades & 70,294 & 70,294 & 70,294 & 70,294 & & 70,294 \\
\hline EF año & $\mathrm{Si}$ & $\mathrm{Si}$ & No & No & No & No \\
\hline EF año-industria & No & No & $\mathrm{Si}$ & No & $\mathrm{Si}$ & $\mathrm{Si}$ \\
\hline EF año-país & No & No & No & $\mathrm{Si}$ & $\mathrm{Si}$ & $\mathrm{Si}$ \\
\hline EF producto-país & $\mathrm{Si}$ & $\mathrm{Si}$ & $\mathrm{Si}$ & $\mathrm{Si}$ & No & $\mathrm{Si}$ \\
\hline
\end{tabular}


Tabla A4. Cambio en la calidad a nivel producto

\begin{tabular}{|c|c|c|c|c|c|c|}
\hline & \multicolumn{6}{|c|}{ Calidad } \\
\hline & (1) & $(2)$ & $(3)$ & (4) & $(5)$ & $(6)$ \\
\hline \multicolumn{7}{|l|}{ Panel A: MCO } \\
\hline Post & $\begin{array}{c}-0.579^{* * *} \\
(0.016)\end{array}$ & $\begin{array}{c}-0.589^{* * *} \\
(0.017)\end{array}$ & $\begin{array}{c}-0.583^{* * *} \\
(0.017)\end{array}$ & $\begin{array}{c}-0.586^{* * *} \\
(0.017)\end{array}$ & $\begin{array}{c}-0.597^{* * *} \\
(0.017)\end{array}$ & $\begin{array}{c}-0.582^{* * *} \\
(0.017)\end{array}$ \\
\hline Observaciones & 38,220 & 38,220 & 38,220 & 38,220 & 38,220 & 38,220 \\
\hline R-cuadrado & 0.059 & 0.057 & 0.056 & 0.057 & 0.053 & 0.056 \\
\hline Productos & 7,425 & 7,425 & 7,425 & 7,425 & 7,425 & 7,425 \\
\hline \multicolumn{7}{|l|}{ Panel B: VI } \\
\hline Post & $\begin{array}{c}-0.567^{* * *} \\
(0.016)\end{array}$ & $\begin{array}{c}-0.602^{* * *} \\
(0.017)\end{array}$ & $\begin{array}{c}-0.609^{* * * *} \\
(0.018)\end{array}$ & $\begin{array}{c}-0.603^{* * *} \\
(0.018)\end{array}$ & $\begin{array}{c}-0.606^{* * *} \\
(0.018)\end{array}$ & $\begin{array}{c}-0.618^{* * *} \\
(0.018)\end{array}$ \\
\hline Observaciones & 38,097 & 38,097 & 38,097 & 38,097 & 38,097 & 38,097 \\
\hline R-cuadrado & 0.060 & 0.056 & 0.055 & 0.054 & 0.053 & 0.054 \\
\hline Productos & 7,400 & 7,400 & 7,400 & 7,400 & 7,400 & 7,400 \\
\hline \multicolumn{7}{|c|}{ Panel C: VI. Por año } \\
\hline Año 2000 & $\begin{array}{c}-0.078^{* * *} \\
(0.016)\end{array}$ & $\begin{array}{c}-0.084^{* * *} \\
(0.018)\end{array}$ & $\begin{array}{c}-0.087^{* * *} \\
(0.019)\end{array}$ & $\begin{array}{c}-0.086^{* * *} \\
(0.019)\end{array}$ & $\begin{array}{c}-0.087^{* * *} \\
(0.019)\end{array}$ & $\begin{array}{c}-0.090^{* * *} \\
(0.019)\end{array}$ \\
\hline Año 2001 & $\begin{array}{c}-0.255^{* * *} \\
(0.017)\end{array}$ & $\begin{array}{c}-0.268^{* * *} \\
(0.02)\end{array}$ & $\begin{array}{c}-0.272^{* * *} \\
(0.02)\end{array}$ & $\begin{array}{c}-0.271^{* * *} \\
(0.02)\end{array}$ & $\begin{array}{c}-0.273^{* * *} \\
(0.021)\end{array}$ & $\begin{array}{c}-0.277^{* * *} \\
(0.021)\end{array}$ \\
\hline Año 2002 & $\begin{array}{c}-1.099 * * * \\
(0.027)\end{array}$ & $\begin{array}{c}-1.171^{* * *} \\
(0.03)\end{array}$ & $\begin{array}{c}-1.184^{* * *} \\
(0.03)\end{array}$ & $\begin{array}{c}-1.175^{* * *} \\
(0.03)\end{array}$ & $\begin{array}{c}-1.179^{* * *} \\
(0.03)\end{array}$ & $\begin{array}{c}-1.199^{* * *} \\
(0.031)\end{array}$ \\
\hline Año 2003 & $\begin{array}{c}-0.612^{* * *} \\
(0.022)\end{array}$ & $\begin{array}{c}-0.648^{* * *} \\
(0.024)\end{array}$ & $\begin{array}{c}-0.656^{* * *} \\
(0.025)\end{array}$ & $\begin{array}{c}-0.649^{* * *} \\
(0.025)\end{array}$ & $\begin{array}{c}-0.654^{* * *} \\
(0.025)\end{array}$ & $\begin{array}{c}-0.667^{* * *} \\
(0.026)\end{array}$ \\
\hline Año 2004 & $\begin{array}{c}-0.354^{* * *} \\
(0.021)\end{array}$ & $\begin{array}{c}-0.374^{* * *} \\
(0.023)\end{array}$ & $\begin{array}{c}-0.380^{* * *} \\
(0.024)\end{array}$ & $\begin{array}{c}-0.374^{* * *} \\
(0.024)\end{array}$ & $\begin{array}{c}-0.377^{* * *} \\
(0.024)\end{array}$ & $\begin{array}{c}-0.388^{* * *} \\
(0.025)\end{array}$ \\
\hline Observaciones & 38,097 & 38,097 & 38,097 & 38,097 & 38,097 & 38,097 \\
\hline R-cuadrado & 0.101 & 0.094 & 0.093 & 0.093 & 0.090 & 0.091 \\
\hline Productos & 7,400 & 7,400 & 7,400 & 7,400 & 7,400 & 7,400 \\
\hline
\end{tabular}

